\title{
SUPPRESSION OF SECONDARY ELECTRON EMISSION USING TRIANGULAR GROOVED SURFACE IN THE ILC DIPOLE AND WIGGLER MAGNETS*
}

\author{
L. Wang", K. Bane, C. Chen, T. Himel, M. Munro, M. Pivi, T. Raubenheimer, G. Stupakov \\ SLAC, CA 94025, USA
}

\begin{abstract}
The development of an electron cloud in the vacuum chambers of high intensity positron and proton storage rings may limit machine performance. The suppression of electrons in a magnet is a challenge for the positron damping ring of the International Linear Collider (ILC) as well as the Large Hadron Collider. Simulation show that grooved surfaces can significantly reduce the electron yield in a magnet. Some of the secondary electrons emitted from the grooved surface return to the surface within a few gyrations, resulting in a low effective secondary electron yield (SEY) of below 1.0 A triangular surface is an effective, technologically attractive mitigation with a low SEY and a weak dependence on the scale of the corrugations and the external magnetic field. A chamber with triangular grooved surface is proposed for the dipole and wiggler sections of the ILC and will be tested in KEKB in 2007. The strategy of electron cloud control in ILC and the optimization of the grooved chamber such as the SEY, impedance as well as the manufacturing of the chamber, are also discussed.
\end{abstract}

\section{INTRODUCTION}

The updated baseline design of the ILC Damping Rings consists of one $6 \mathrm{~km}$ ring for both the electron and positron beams. There is a short bunch spacing of 3 6 $\mathrm{ns}$ and small beam pipe aperture of $20 \mathrm{~mm}$ in ILC, which causes more electron cloud in general. The electron cloud in the field free region can be suppressed by solenoids, which have been well demonstrated in the B-factories as a good mitigation [1]. There are hundreds of meters of wiggler sections in the ILC and the remained electron cloud in the dipole and wiggler magnets can cause beam instability [2] and beam loss. The electron cloud in the DAFNE wiggler is suspected to be the source of instability there [3].

Clearing electrodes with various designs are proposed to kill the secondary electrons in a dipole magnet in the SPS, PEPII and KEKB [4-7]. Increasing the surface roughness is another way to reduce the effective SEY. In this case, the effective SEY is reduced because the secondaries have multiple collisions with the surface where, at low energy, the SEY $<<1$. There are different suppression machnisnism of the surface roughness on the secondary emission in the field free region and magnet. A dipole magnetic field attenuates the photoelectron emission from the surface by more than two orders of magnitude when the magnetic field is aligned parallel to the surface [8]. A grooved surface is incorporated within the multipacting surface in superconducting cavities to disturb electron trajectories and prevent resonant electron production [9]. The suppression of the SEY with triangular and rectangular surfaces in a magnetic field has been simulated without the detailed variation of parameters [10, 11]. Simulation shows that triangular grooved surfaces can significantly reduce the electron yield in a magnet [12]. Some of the secondary electrons emitted from the grooved surface return to the surface within few gyrations, resulting in a low effective secondary electron yield (SEY) below 1.0. The former simulation shows that triangular grooves are not as effective [10]. A chamber with a triangular grooved surface is proposed for the dipole and wiggler sections of the ILC. A test chamber will be installed in KEKB LER. This paper discusses the design of the triangular grooved chamber, including its effects of SEY and impedance.

\section{SUPPRESSION OF SEY}

Both triangular and rectangular grooved surfaces can reduce the effective SEY. However, a triangular surface has a weak dependence on the scale of the corrugations and the external magnetic field.

The geometry of a triangular surface is shown in Figure 1 where $W$ is the period of the surface. If an electron hits the surface and a secondary is emitted, the secondary may complete a partial gyration and then hit the surface again with a low SEY due to its low energy. The probability of a secondary electron returning to the surface depends on the angle $\alpha$. The period of the electron's gyration $2 \pi m_{0} \gamma / e B_{0}$ is $0.179 \mathrm{~ns}$ in a 0.2 Tesla magnetic field, while the bunch spacing in the ILC damping ring and Bfactories ranges from 3 to $8 \mathrm{~ns}$. Thus, the secondary electrons can hit the surface dozens of times between beam passages. This is similar to the mechanism for suppressing the electrons with a weak solenoid in a field free region. The electrons' orbits in Figure 1 clearly show the trapping mechanism.

Figure 2 shows the simulated effective SEY of a triangular surface with different $W$ and $\alpha$ in a dipole magnet. The simulated effective SEY is very sensitive to the angle $\alpha$. The SEY decreases from 1.3 to 0.4 when $\alpha$ increases from $65^{\circ}$ to $80^{\circ}$. The effective SEY has a weak dependence on $W$. Moreover, at large $W$, it reaches saturation with a minimum SEY. The saturation of the SEY at large $W$ offers more opportunity for optimization of the grooved geometry. In general, a mm-scale surface is easier to manufacture. For comparison, note that the gyration motion radius of an electron with energy at peak SEY ( $330 \mathrm{eV}$ here) is $0.306 \mathrm{~mm}$.

Figure 3 shows the simulated effective SEY of

*Work supported by the U.S. Department of Energy under contract DE-AC02-76SF00515

\# email address: wanglf@slac.stanford.edu

Presented at Particle Accelerator Conference (PAC 2007), 6/25/2007-6/29/2007, Albuquerque, NM, USA 
triangular surface with different $\alpha$ in a 1.6 Tesla dipole field, which is the peak field of the ILC wiggler. A constant $W=1.89 \mathrm{~mm}$ is used here. Comparison of Figure 3 with Figure 2 shows that the effect of the magnetic field is negligible at large $W$.

Another simulation shows triangular grooves are less effective in dipole field [10]. For example, a triangular surface with $\alpha=70^{\circ}$ reduces the peak SEY from 1.74 to 1.5 , while it is below 1.0 in our study. The simulation of electron cloud build-up shows that the electron cloud density is reduced by a factor of 200 (effective SEY about 1.0) in a 0.2 Tesla dipole magnet with triangular grooved surface $\alpha=75^{\circ}$ [13]. Considering the very different approaches are used, it is good agreement with our calculation.

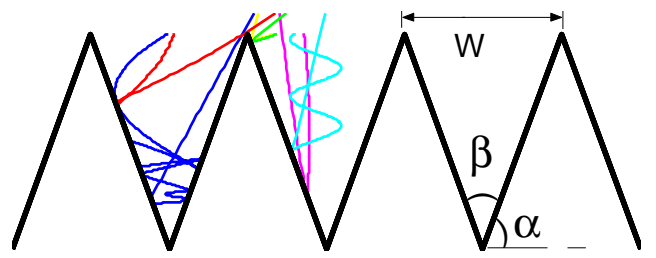

Figure 1: Triangular surface $(\alpha+\beta / 2=\pi / 2)$.

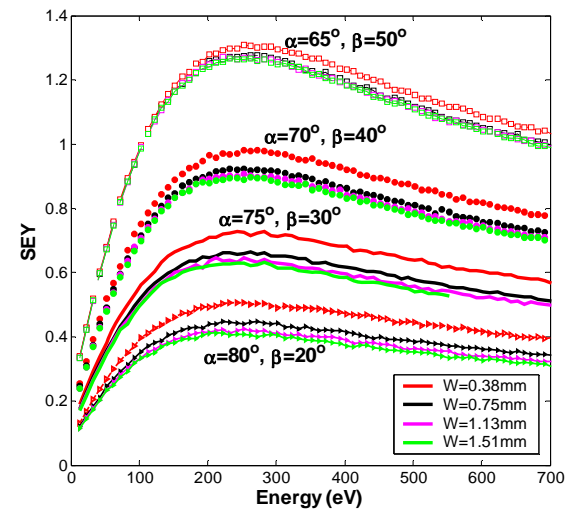

Figure 2: Effective SEYs of isosceles triangular surfaces with $\alpha=65^{\circ}, 70^{\circ}, 75^{\circ}$, and $80^{\circ}$ in a magnetic field of 0.2 Tesla. For each $\alpha$, the SEYs with $W=0.38 \mathrm{~mm}, 0.75 \mathrm{~mm}$, $1.13 \mathrm{~mm}$ and $1.51 \mathrm{~mm}$ are calculated. The parameters of SEY are $\delta_{\max }=1.74$ and $E_{\max }=330 \mathrm{eV}$.

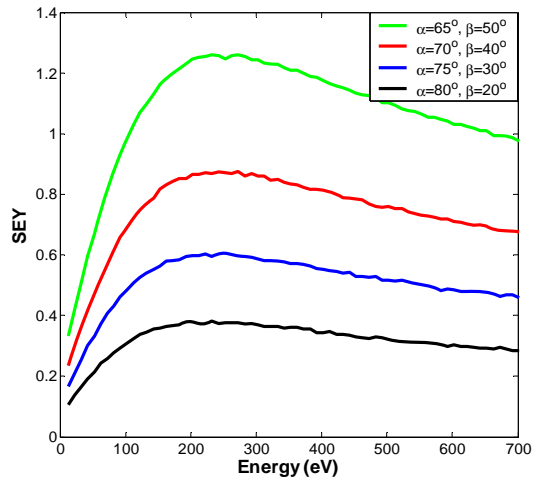

Figure 3: Effective SEYs from isosceles triangular surfaces with $\alpha=65^{\circ}, 70^{\circ}, 75^{\circ}$, and $80^{\circ}$ in a magnetic field of 1.6 Tesla. The period $W=1.89 \mathrm{~mm}$. The parameters of SEY are $\delta_{\max }=1.74$ and $E_{\max }=330 \mathrm{eV}$.

\section{DESIGN OF THE TEST CHAMBER}

A chamber with a triangular grooved surface is going to be tested in KEKB this year. Figure 4 shows the transverse distribution of the electron cloud in the dipole and quadrupole magnets of ILC positron damping ring using the simulation program CLOUDLAND. Two multipacting strips near the center are clearly visible in the dipole magnet. The width of the multipacting region where the grooved surface would be required is only 10 $\mathrm{mm}$ and the required grooved surface is only $15 \%$ of the total surface. The width of the multipacting strips depends on the beam current, magnetic field and aperture of the beam chamber. Simulations of the electron cloud effect with various design parameters can illustrate the multipacting width. Generally, there is a larger width of multipacting region for a higher beam current. Thus, we should leave a safety margin for the possible upgrade of the machine when we choose the width of the grooved surface.

Figure 5 shows a sketch of the test chamber in a dipole magnet. The bottom and top of the beam pipe is covered by the triangular grooved surface. An electron collector is attached at the top of the chamber. Electrons go through the holes between the beam pipe and collector to reach the multi-strips anode (collector) in order to measure the electron's lateral distribution. There are two meshes of a shield and a retarding grid between the beam pipe and strips.

An ideal triangular surface is desired in our technique in order to trap the secondary electrons. In principle, machining could produce the sharp corners, but technically, it is a very difficult and an expensive approach, compared to abrasive blasting or chemical etching. The grooved surface can also be made by the extrusion of a relatively soft material, such as aluminum or copper. TiN-coated aluminum will be used to make a low SEY triangular surface for the test.

During fabrication, the tip of the triangle is likely to be rounded and a rounded tip will not reduce the SEY as well. Figure 6 shows a triangular surface with/without rounded tips and Fig. 7 shows the effective SEYs of these geometries. The radius of the rounded tip $R_{t i p}$ is $0.2 \mathrm{~mm}$. The SEY increases due to the rounded tips, but it still can significantly lower the SEY. The effect of round tips can be mitigated by choosing a large $W$ comparing with $R_{t i p}$. Due to the sensitivity of the geometry, the fabrication error should be well controlled in order to get as small an SEY as expected. (a)

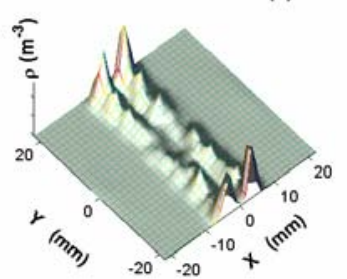

(b)

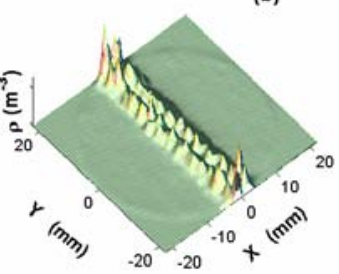

Figure 4: Transverse distribution of electron cloud in a dipole magnet (a) and wiggler magnet (b). 


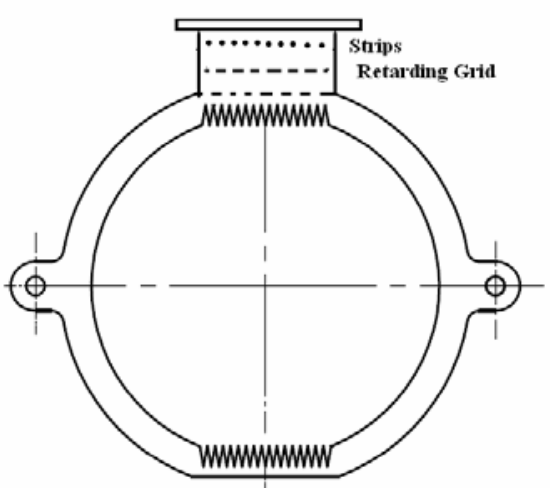

Figure 5: Sketch of a test chamber in dipole magnet. The surface is partially covered by the triangular grooved surface at the top and bottom to suppress the electron multipacting there. The material of the grooved surface is aluminium and is coated with TiN.

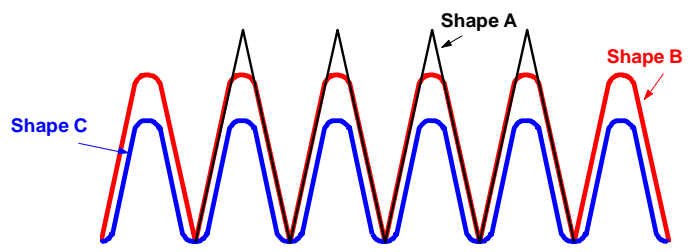

Figure 6: Triangular groove with different tips

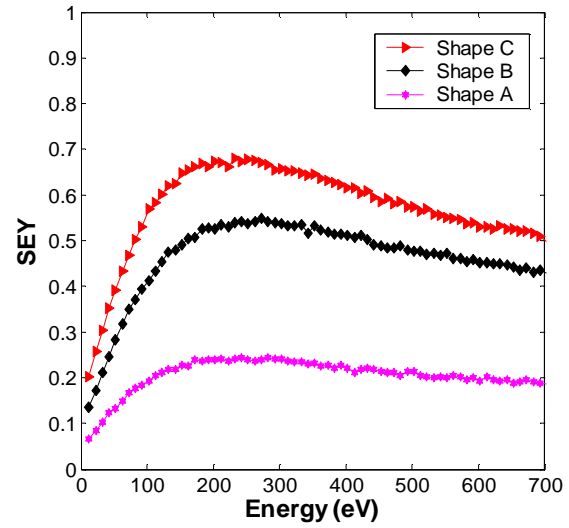

Figure 7: Effective SEYs of a triangular surface with different tips shown in Figure $6\left(R_{t i p}=0.2 \mathrm{~mm}, W=2 \mathrm{~mm}\right.$, $\alpha=80^{\circ}$ ) in a magnetic field of 0.2 Tesla. The SEY parameters are $\delta_{\max }=1.2$ and $E_{\max }=330 \mathrm{eV}$.

\section{IMPEDANCE ENHANCEMENT}

The energy loss induced by the electromagnetic field in the wall in the small skin depth approximation is proportional to the square of the magnetic field on the metal surface. Therefore, the enhancement $\eta$ of the resistive wall wake effect (both transverse and longitudinal) for the finned beam pipe, compared to a normal beam pipe, can be written as

$$
\eta=\frac{\int H^{2} d s}{H_{0}^{2} W}
$$

where $H$ is the magnetic field of the beam on the surface of the metal, $H_{0}$ the magnetic field in the case of a flat (non-grooved) surface, and the integration follows the grooved surface over one period in a plane of constant $z$. The magnetic field can be represented as $\mathbf{H}=\hat{z} \times \nabla \varphi$, with $\hat{z}$ the unit vector in $z$ and the magnetic potential $\varphi$ satisfying the two-dimensional Laplace equation $\nabla^{2} \varphi=0$.

Note that using the Laplace equation for the magnetic field is valid for frequencies $\omega$ such that $c / \omega>>W$; for example, for $W \sim 3 \mathrm{~mm}$ this means $\omega \leq 2 \pi \cdot 10^{11} \mathrm{~Hz}$.

The impedance enhancement of the triangular groove with sharp tips is $\eta=1 / \cos \alpha$ [12]. The impedance enhancement factor is large at large angle $\alpha$. For the geometry with round tips, the impedance enhancement is calculated using numerical calculation method. An adaptive method is applied to reduce the calculation time and improve the accuracy [14]. The results are shown in Figure 8. The impedance enhancement factor increases linearly with angle $\alpha$ in the region we are interested in and it is smaller than 2.0. The width of the multipacting region where the grooved surface would be required is only 10 $\mathrm{mm}$ and the required grooved surface is only $15 \%$ of the total surface. Therefore, the overall impedance enhancement due to the grooved surface is small.

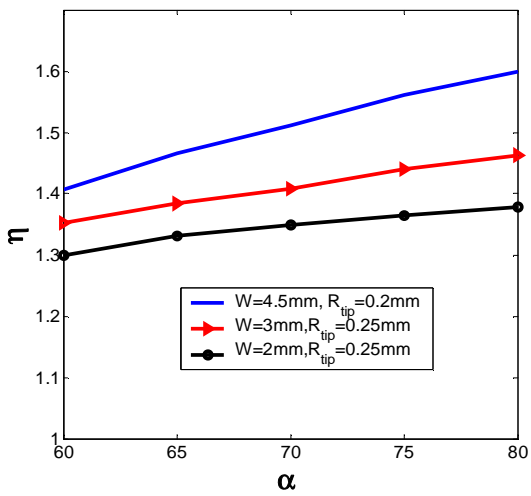

Figure 8: Impedance enhancement factor for the triangular grooved surface with round tips.

\section{REFERENCES}

[1]H. Fukuma, CERN-2005-001, Proceedings of ECLOUD'04

[2] M. Pivi, et. al., Proceedings of EPAC06, 2958 (2006)

[3]F. Zimmermann, et. al., CLIC Note 650, EUROTEVREPORT 2006-002-1

[4] P.McIntyre and A.Sattarov, Proceedings of PAC05, 2971 (2005)

[5] L. Wang, et. al., Proceedings of EPAC06, 1489 (2006)

[6] M. Pivi, et. al., see these Proceedings.

[7] Y. Suetsugu and H. Fukuma, private communications.

[8] V.V. Anashin, et al., Vacuum 60 (2001) 255

[9] H. Padamsee, et. al. IEEE Trans. Magn.17,947 (1981).

[10] G. Stupakov and M. Pivi, SLAC-TN-04-045(2004).

[11] M. Pivi, et al., in Proceedings of PAC2005 p205

[12] L. Wang, T. O. Raubenheimer and G. Stupakov, Nucl. Instrum. Meth. A571:588-598, 2007

[13] M. Venturini, et. al., these Proceedings

[14] L. Wang, these Proceedings. 